\title{
A novel solution algorithm for nonlinearly loaded transmission lines inside resonating enclosures
}

\author{
R. Rambousky ${ }^{1}$, S. Tkachenko ${ }^{2}$, and J. Nitsch ${ }^{2}$ \\ ${ }^{1}$ Bundeswehr Research Institute for Protective Technologies and NBC Protection (WIS), Munster, Germany \\ ${ }^{2}$ Otto-von-Guericke University Magdeburg, Magdeburg, Germany \\ Correspondence to: R. Rambousky (ronald.rambousky@ieee.org)
}

Received: 28 December 2013 - Revised: 15 March 2014 - Accepted: 22 April 2014 - Published: 10 November 2014

\begin{abstract}
Nonlinearly loaded lossless transmission lines inside a rectangular cavity are studied using the left- and righthand Green's functions of the problem in time domain. These Green's functions are developed for a transmission line with quasi-matched loads. This ensures Green's functions of a short duration. Therefore, the amount of frequency data necessary to obtain time-domain Green's functions is quite limited. The time-domain Green's functions are finally convolved with the left- and right-hand line voltages. With this technique it is possible to treat arbitrarily loaded transmission lines in resonators. An example is presented to demonstrate the applicability of this technique to a transmission line with a simple diode as nonlinear load.
\end{abstract}

\section{Introduction}

Interconnecting cables play a major role in Electromagnetic Compatibility (EMC). These cables do not only transport information signals and energy in electronic systems, but they also act as effective collectors for unintended electromagnetic energy. Electronic components usually possess basic EMC hardness but often they are integrated in metallic housings and interconnected with other components. Therefore, the resulting configuration may differ significantly from the EMC test configuration of the single electronic component. Resonance effects of the housing, cable coupling in cable harnesses or nonlinear effects (e.g. demodulation) may effect electromagnetic compatibility issues and therefore the reliable operation of the electronic system.

Shielded rooms are designed to provide the electronic devices as a whole a sufficient electromagnetic protection. Since the shielding factor decreases for non-ideal screens with increasing frequencies, an intentional electromagnetic disturbance of a globally protected system will be expected more at the higher frequencies. If nonlinearities are excited by higher frequencies, then they also generate lowerfrequency components in the noise spectrum by demodulation effects which may cause a number of problems for the electronic system, like, e.g., distortion of useful electronic signals, shift of operating points of transistor cascades of electronic circuits, or generation of dynamic instabilities in complex electronic systems.

Every electromagnetic field excitation of a shielded room, like a rectangular resonator, may generate resonance frequencies which are closely adjacent, in particular in the high frequency spectrum. Therefore, two-tone signals can be produced (Krauthäuser et al., 2002) that lead by field to cable coupling to demodulation effects in the current spectra in non-linear terminations. Other mechanisms which may also cause a two-tone high-frequency field inside the resonator is an exterior field excitation which enters the resonator through an aperture or is generated by an interior small antenna inside the resonator (Rambousky et al., 2013).

In this work the influence of nonlinear loads on cables (transmission lines) concerning transmitted current spectra is investigated. Transmission lines with nonlinear loads over PEC ground in free space show the expected demodulation and intermodulation effects. In resonators additionally there is a strong coupling of the transmission line with the resonance structure of the resonator and therefore the resulting spectra are much more comprehensive. In particular the resulting electromagnetic fields in the resonator increase significantly when the excitation frequency coincide with the resonance frequencies of the resonator. 


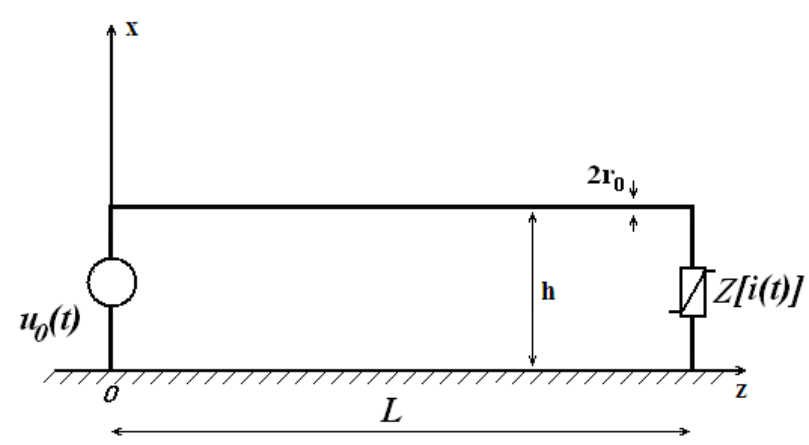

Figure 1. Geometry of the conductor above conducting ground.

Consider a classical transmission line which is fed/terminated at both ends by two active/passive sources $u_{0}(t)$ and $u_{r}(t)$, respectively. If one further assumes that the two corresponding left- and right-hand Green's functions $g_{l}(t)$ and $g_{r}(t)$ in time-domain are known then the current $i_{L}(t)$ in time-domain at the end of the transmission line at $z=L$ can be expressed as a sum of convolution integrals of these Green's functions and the two (voltage) sources:

$$
\begin{aligned}
i_{L}(t) & =\int_{-\infty}^{t} g_{l}(L, t-\tau) u_{0}(\tau) d \tau \\
& +\int_{-\infty}^{t} g_{r}(L, t-\tau) u_{r}(\tau) d \tau
\end{aligned}
$$

Thus, with the knowledge of these two Green's functions one can calculate the current $i_{L}(t)$, for linear as well as for nonlinear terminations of the transmission line. In this paper, the emphasis is laid on estimating these Green's functions.

\section{Nonlinearly loaded transmission lines in free space above conducting ground}

Consider first a linearly terminated transmission line above a perfectly conducting ground in the Agrawal form in free space (for the geometry see Fig. 1). The solution for this problem is known (Liu and Tesche, 1976) for the current at the end of the line $(z=L)$ and can be represented as

$I(L, \omega)=U_{0}(\omega) Y_{l}(L, \omega)+U_{r}(\omega) Y_{r}(L, \omega)$.

Here $U_{0}(\omega)$ and $U_{r}(\omega)$ are the left- and right-hand sources of the conductor, respectively. The functions $Y_{l}(L, \omega)$ and $Y_{r}(L, \omega)$ are the corresponding complex admittance functions at the end $(z=L)$ of the line. They are given in frequency domain by the expressions

$Y_{l}(L, \omega)=\frac{2}{Z_{C}} \sum_{m=0}^{\infty} \exp (-\jmath(2 m+1) k L)$ and

$Y_{r}(L, \omega)=\frac{1}{Z_{C}}\left[1+2 \sum_{m=1}^{\infty} \exp (-\jmath 2 m k L)\right]$,

respectively. The quantity $Z_{C}$ denotes the characteristic impedance of the line, $k=\omega / c_{0}$ is the wave number and $J \equiv \sqrt{-1}$ the imaginary unit.

An inverse Fourier transformation of Eqs. (3) and (4) into time-domain yields

$y_{l}(L, t)=\frac{2}{Z_{C}} \sum_{m=0}^{\infty} \delta\left(t-(2 m+1) \frac{L}{c_{0}}\right)$

and

$y_{r}(L, t)=\frac{1}{Z_{C}}\left[\delta(t)+2 \sum_{m=1}^{\infty} \delta\left(t-2 m \frac{L}{c_{0}}\right)\right]$.

The dirac delta function is denoted by $\delta(t)$ and $c_{0}$ is the speed of light in vacuum. The convolution integral of Eq. (1) can simply be calculated and results in:

$$
\begin{gathered}
i(L, t)=\frac{2}{Z_{C}} \sum_{m=0}^{\infty} u_{0}\left(t-(2 m+1) \frac{L}{c_{0}}\right) \\
+\frac{1}{Z_{C}}\left[u_{r}(t)+2 \sum_{m=1}^{\infty} u_{r}\left(t-2 m \frac{L}{c_{0}}\right)\right]
\end{gathered}
$$

This is the general solution in time-domain. Here the restriction to linear loads can be abandoned, and Eq. (7) now is valid for both, linear as well as nonlinear terminations. Assuming for the source at the right side of the conductor the relation

$$
-\frac{u_{r}(t)}{Z_{C}}=\frac{i(L, t) Z(i(L, t))}{Z_{C}}=: \tilde{i}(t)
$$

one obtains from Eq. (7)

$$
\begin{array}{r}
i(L, t)+\tilde{i}(t)=\frac{2}{Z_{C}} \sum_{m=0}^{\infty} u_{0}\left(t-(2 m+1) \frac{L}{c_{0}}\right) \\
-2 \sum_{m=1}^{\infty} \tilde{i}\left(t-2 m \frac{L}{c_{0}}\right) .
\end{array}
$$

If the voltage-current characteristic of the nonlinear load and the driving source $u_{0}(t)$ for $t \geq 0$ are known, Eq. (9) can be solved, in most cases numerically. Noting that the right-hand side of Eq. (9) depends only on past values of $t$, this equation may be solved for the unknown current $i(L, t)$ for later times using a standard root-finding algorithm.

\section{Transmission line with nonlinear load inside a rectan- gular cavity}

In this case the transmission line is positioned symmetrically inside the cavity (see Fig. 2). Before the actual problem 


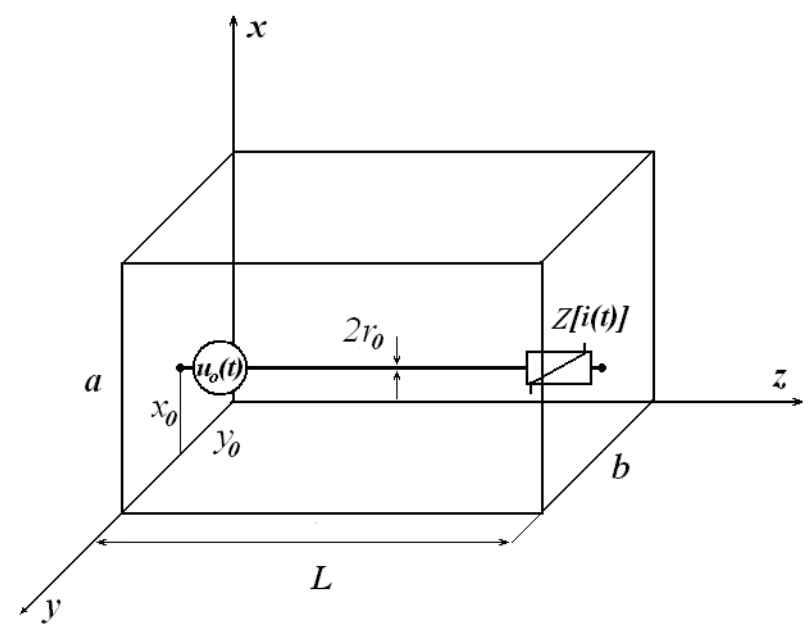

Figure 2. Schematic representation of the TL inside the cavity.

is treated, some important preliminary remarks are made. First, concerning the notations and results of a TL inside a rectangular resonator, reference is made to the publications (Tkachenko et al., 2011, 2013). The second observation relates to a numerical problem. Since a lossless transmission line is considered inside a cavity with a very high Q-value it is expected that the response of a once excited transmission line will exceed many transit times in duration. If, in addition, very high frequencies are required, one may end up not only in a computer-storage problem, but also needs very long computation time. Therefore, it would be desirable to reduce the duration of the Green's functions (here represented by the admittance functions) to only a few transit times. This, however, is possible to achieve, if the TL is matched. Following this idea, it is aimed to have a well matched line when computing the Green's functions (admittance functions). This goal is achieved in the following novel analysis.

For the moment the calculations take place in the frequency domain. During this time the terminations of the line are assumed to be linear. The explicit dependency of the frequencies, however, is suppressed in the quantities. In the rectangular resonator the admittance functions are given by:

$Y_{l}(z):=\frac{J k}{\eta_{0} L} \sum_{n_{3}=0}^{\infty} \frac{\epsilon_{n_{3}, 0} \cos \left(\frac{n_{3} \pi z}{L}\right)}{\left(k_{v z}^{2}-k^{2}\right) S}$

and

$Y_{r}(z):=\frac{J k}{\eta_{0} L} \sum_{n_{3}=0}^{\infty} \frac{\epsilon_{n_{3}, 0}(-1)^{n_{3}} \cos \left(\frac{n_{3} \pi z}{L}\right)}{\left(k_{v z}^{2}-k^{2}\right) S}$

Then the current along the line is expressed through

$I(z)=U_{0} Y_{l}(z)+U_{r} Y_{r}(z)=U_{0} Y_{l}(z)-Z I(L) Y_{r}(z)$

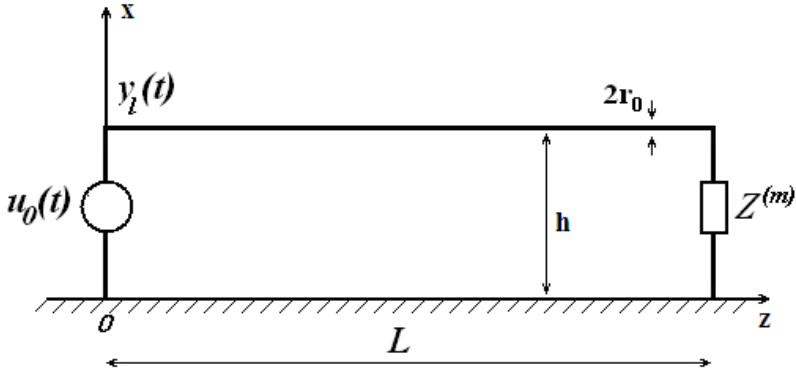

Figure 3. Matched TL inside the resonator with left-hand source.

and thus at the end of the line $(z=L)$ one has

$I(L)=\frac{U_{0} Y_{l}(L)}{1+Z Y_{r}(L)}$

The impedance $Z$ is now decomposed into two partial impedances, of which one $\left(Z^{(m)}\right)$ corresponds to the characteristic impedance (Djordjevic et al., 1986):

$Z=Z^{(m)}+Z^{(n l)} \quad$ with $\quad Z^{(m)} \equiv Z_{C}$

Thereafter, an auxiliary circuit with $Z^{(m)}$ as terminating impedance as shown in Fig. 3 is examined. For this circuit one has:

$I(z)=U_{0} Y_{l}(z)-Z^{(m)} I(L) Y_{r}(z)$

or solved for the current at the end of the line

$I(L)=\frac{U_{0} Y_{l}(L)}{1+Z^{(m)} Y_{r}(L)}$

Inserting Eq. (16) into Eq. (15) leads to

$I(z)=U_{0} \tilde{Y}_{l}^{\left(Z^{(m)}\right)}(z)$

with the new admittance function

$\tilde{Y}_{l}^{\left(Z^{(m)}\right)}(z):=Y_{l}(z)-\frac{Z^{(m)} Y_{l}(L) Y_{r}(z)}{1+Z^{(m)} Y_{r}(L)}$

In the next step, consider the same line from which the source $U_{0}(\omega)$ was removed. A source (active or passive) was instead inserted on the right side. The impedance at the end of the line is again $Z^{(m)}$ (see Fig. 4). Then, the following applies:

$I(z)=U_{r} Y_{r}(z)-Z^{(m)} I(L) Y_{r}(z)$

or at the point $z=L$

$I(L)=\frac{U_{r} Y_{r}(L)}{1+Z^{(m)} Y_{r}(L)}$

Substituting Eq. (20) into Eq. (19) yields the current for the right-hand source

$I(z)=U_{r} \tilde{Y}_{r}^{\left(Z^{(m)}\right)}(z)$ 


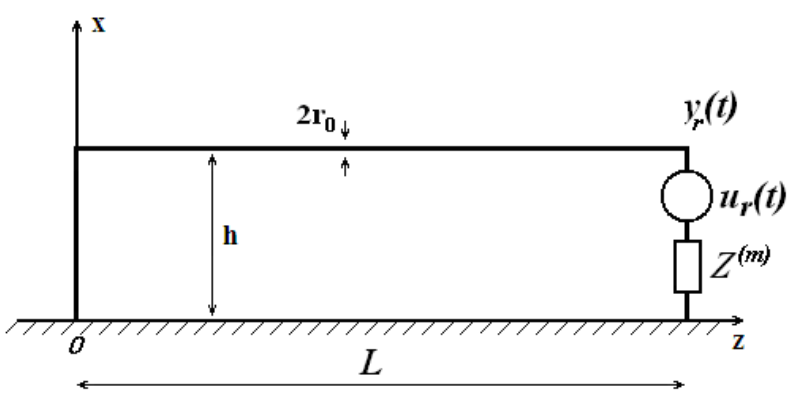

Figure 4. Matched TL inside the resonator with right-hand source.

with

$\tilde{Y}_{r}^{\left(Z^{(m)}\right)}(z):=Y_{r}(z)-\frac{Z^{(m)} Y_{r}(L) Y_{r}(z)}{1+Z^{(m)} Y_{r}(L)}$

With these new admittance functions (18) and (22) the above calculation is repeated for the following configuration depicted in Fig. 5. Now one obtains:

$I(z)=U_{0} \tilde{Y}_{l}^{\left(Z^{(m)}\right)}(z)-Z^{(n l)} I(L) \tilde{Y}_{r}^{\left(Z^{(m)}\right)}(z)$

In Eq. (23) $U_{r}$ is assumed to be a passive source. Thus, the current at the end of the line results in:

$I(L)=\frac{U_{0} \tilde{Y}_{l}^{\left(Z^{(m)}\right)}(L)}{1+Z^{(n l)} \tilde{Y}_{r}^{\left(Z^{(m)}\right)}(L)}$

Using the renormalized admittance functions (18) and (22) in Eq. (24) it follows the expected result

$$
I(L)=\frac{U_{0} Y_{l}(L)}{1+\left(Z^{(m)}+Z^{(n l)}\right) Y_{r}(L)}
$$

But this is exactly the Eq. (13), taking into account the decomposition (14). This demonstrates that a given impedance can be decomposed into two parts where $Z^{(m)}$ can be suitably selected. In general, this will be the characteristic impedance of the line.

Now, by an inverse Fourier transform (23) is transformed into time-domain $(z=L)$.

$i_{L}(t)=u_{0}(t) * \tilde{y}_{l}^{\left(Z^{(m)}\right)}(L, t)+u_{r}(t) * \tilde{y}_{r}^{\left(Z^{(m)}\right)}(L, t)$

The convolution integrals in Eq. (26) are indicated by ' $*$ '. In explicit form this reads:

$$
\begin{aligned}
i_{L}(t) & =\int_{-\infty}^{t} \tilde{y}_{l}^{\left(Z^{(m)}\right)}(L, t-\tau) u_{0}(\tau) d \tau \\
& +\int_{-\infty}^{t} \tilde{y}_{r}^{\left(Z^{(m)}\right)}(L, t-\tau) u_{r}(\tau) d \tau
\end{aligned}
$$

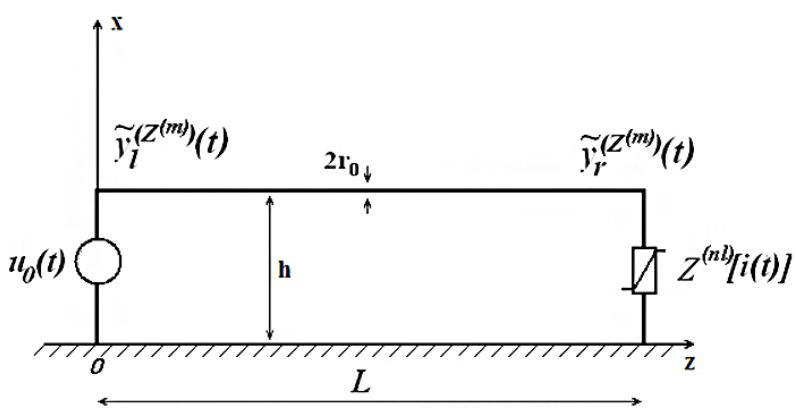

Figure 5. TL is fed by $U_{0}$ and terminated by $Z^{(n l)}$; current is estimated with the aid of renormalized admittance functions.

Here one clearly recognizes that the $\tilde{y}$-functions are the time-domain Green's functions, i.e., the responses of the terminated line in the resonator due to impulse excitations. Remember, these $\tilde{y}$-functions have been determined in frequency-domain for the linear part of the TL only (see Figs. 3 and 4). Equation (27) now is valid in time-domain. Therefore, also nonlinear loads can be chosen and there is no need to recompute frequency-domain data again if the excitation wave form or the (nonlinear) loads are changed.

Since causal functions are assumed $\left(u_{0}(t) \equiv 0\right.$ for $\left.t \leq 0\right)$, and in the time domain the constraint to linear termination impedances now is abandoned, one arrives at the temporary result:

$$
\begin{array}{r}
i_{L}(t)=\int_{0}^{t} \tilde{y}_{l}^{\left(Z^{(m)}\right)}(L, t-\tau) u_{0}(\tau) d \tau \\
-\int_{0}^{t} \tilde{y}_{r}^{\left(Z^{(m)}\right)}(L, t-\tau) Z^{(n l)}\left(i_{L}(\tau)\right) i_{L}(\tau) d \tau
\end{array}
$$

In general, this equation has to be solved numerically. For this purpose it is advisable to rewrite Eq. (28) a little bit to

$$
\begin{array}{r}
i_{L}(t)=i_{0 L}(t) \\
-\int_{0}^{t-\Delta} \tilde{y}_{r}^{\left(Z^{(m)}\right)}(L, t-\tau) Z^{(n l)}\left(i_{L}(\tau)\right) i_{L}(\tau) d \tau \\
-\int_{t-\Delta}^{t} \tilde{y}_{r}^{\left(Z^{(m)}\right)}(L, t-\tau) Z^{(n l)}\left(i_{L}(\tau)\right) i_{L}(\tau) d \tau
\end{array}
$$

with the abbreviation

$i_{0 L}(t):=\int_{0}^{t} \tilde{y}_{l}^{\left(Z^{(m)}\right)}(L, t-\tau) u_{0}(\tau) d \tau$.

After a change of variables in the second integral of Eq. (29) one arrives at a useful starting formula for numerical 
integration.

$$
\begin{aligned}
& i_{L}(t)+\int_{0}^{\Delta} \tilde{y}_{r}^{\left(Z^{(m)}\right)}(L, \tau) Z^{(n l)}\left(i_{L}(t-\tau)\right) i_{L}(t-\tau) d \tau \\
= & i_{0 L}(t)-\int_{0}^{t-\Delta} \tilde{y}_{r}^{\left(Z^{(m)}\right)}(L, t-\tau) Z^{(n l)}\left(i_{L}(\tau)\right) i_{L}(\tau) d \tau
\end{aligned}
$$

The integral on the left-hand side of Eq. (31) contains $\tilde{y}_{r}^{\left(Z^{(m)}\right)}(L, \tau)$ - the right-hand admittance function - which is considered only over a short time-span $0 \leq \tau \leq \Delta$. During this short time $\Delta$, the line still does not interact with the resonator. It behaves like a line in free space (see Eq. 6). Therefore, in this small period of time around $\left.t=0, \tilde{y}_{r}{ }^{(m)}\right)(L, \tau)$ is represented as a $\delta$-function, or more precisely:

$\tilde{y}_{r}^{\left(Z^{(m)}\right)}(L, \tau)=\frac{1}{2 Z_{C}} \delta(\tau)$ for $\quad-\Delta \leq \tau \leq \Delta$

The $\delta$-function here can be represented as a Gaussianfunction:

$\delta(\tau) \approx \frac{1}{\sqrt{\pi} \tau_{0}} \exp \left(-\frac{\tau^{2}}{\tau_{0}^{2}}\right)$

using $\quad \tau_{0}=0.1 \mathrm{~ns}, \Delta=4 \tau_{0}, \Delta t=\frac{\tau_{0}}{40}$

Now one can perform the integration on the left-hand side of Eq. (31) and obtains

$$
\begin{aligned}
& i_{L}(t)\left\{1+\frac{1}{2 Z_{C}} Z^{(n l)}\left(i_{L}(t)\right) \frac{\int_{-\Delta}^{\Delta} \exp \left(-\frac{\tau^{2}}{\tau_{0}^{2}}\right) d \tau}{\sqrt{\pi} \tau_{0}}\right\} \\
= & i_{0 L}(t)-\int_{0}^{t-\Delta} \tilde{y}_{r}^{\left(Z^{(m)}\right)}(L, t-\tau) Z^{(n l)}\left(i_{L}(\tau)\right) i_{L}(\tau) d \tau
\end{aligned}
$$

For the numerical integration the integral in Eq. (34) can be rewritten as a sum:

$$
\begin{array}{r}
i_{L}\left(t_{m}\right)\left\{1+\frac{1}{2 Z_{C}} Z^{(n l)}\left(i_{L}\left(t_{m}\right)\right) \frac{\int_{-\Delta}^{\Delta} \exp \left(-\frac{\tau^{2}}{\tau_{0}^{2}}\right) d \tau}{\sqrt{\pi} \tau_{0}}\right\} \\
=i_{0 L}\left(t_{m}\right) \\
-\sum_{k=0}^{m-1} \tilde{y}_{r}^{\left(Z^{(m)}\right)}\left(L, t_{m}-k \Delta t\right) Z^{(n l)}\left(i_{L}(k \Delta t)\right) i_{L}(k \Delta t) \Delta t
\end{array}
$$

Here the sum is calculated at temporal sample points $t_{m}=$ $m \Delta t$, where $m=0,1, \ldots, m_{\max }$. Similar to the situation of

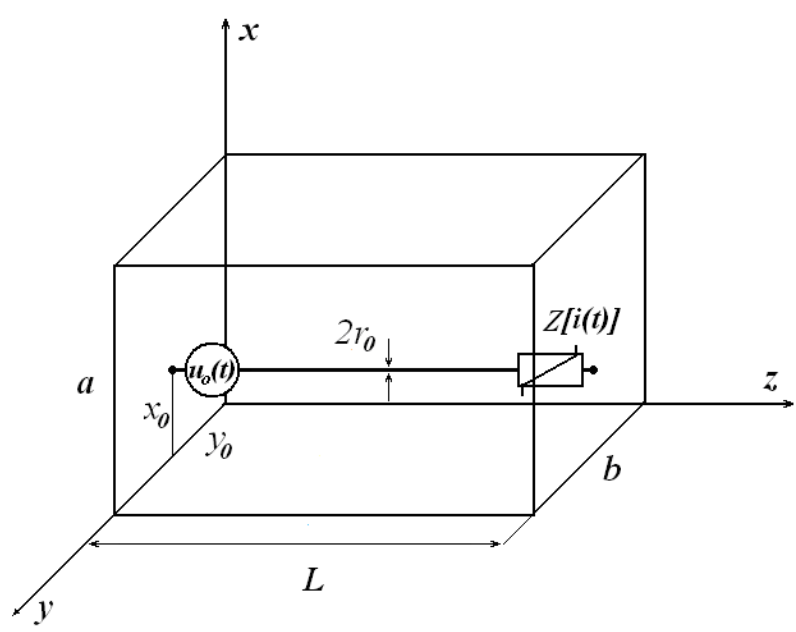

Figure 6. Parameters of the rectangular resonator: $a=30 \mathrm{~cm}, b=$ $53 \mathrm{~cm}, L=79 \mathrm{~cm}$; parameters of the TL: $x_{0}=a / 2, y_{0}=b / 2, r_{0}=$ $1 \mathrm{~mm}$.

Eq. (9), also the right-hand side of Eq. (35) contains expressions of known functions or those that have already been calculated from previous times. Thus, Eq. (35) can be solved for each discrete time $t_{m}$ using a root-finding algorithm.

\section{Generation and discussion of numerical results}

In this section, a Schottky diode is chosen as a nonlinear load and selected results are presented and discussed. The dimensions of the used rectangular resonator and the position of the transmission line in the resonator parallel to the $z$ axis is shown in Fig. 6. In the resonator the TL has a characteristic impedance of $Z_{C}=333 \Omega$. For the following calculations $Z^{(m)}=Z_{C}$ was used. The step size for numerical integration was set to $\Delta t=\tau_{0} / 40$. A diode with the following characteristics

$I(U)=I_{S}\left(\exp \left(U / U_{t}\right)\right) \quad$ or $\quad U(I)=U_{t} \ln \left(1+I / I_{S}\right)$

was regarded and the typical values $U_{t}=25 \mathrm{mV}$ and $I_{S}=$ $1 \mu \mathrm{A}$ were used. A bi-linear approximation of the diode characteristic was choosen and is shown in Fig. 7. The bi-linear approximation of the diode characteristic was generated with the following formula and parameters:

$U(I)=Z(I) \cdot I$

$Z(I)=\left\{\begin{array}{lll}Z_{1} & , \quad I>0 \\ Z_{2} & , & I<0\end{array}\right.$

with $Z_{1}=2 \mathrm{k} \Omega, Z_{2}=0.1 \mathrm{M} \Omega$

In Figs. 8 and 9 the admittance functions $\tilde{y}_{l}^{\left(Z_{C}\right)}(L, t)$ and $\tilde{y}_{r}^{\left(Z_{C}\right)}(L, t)$ are shown in different time scales, respectively. 


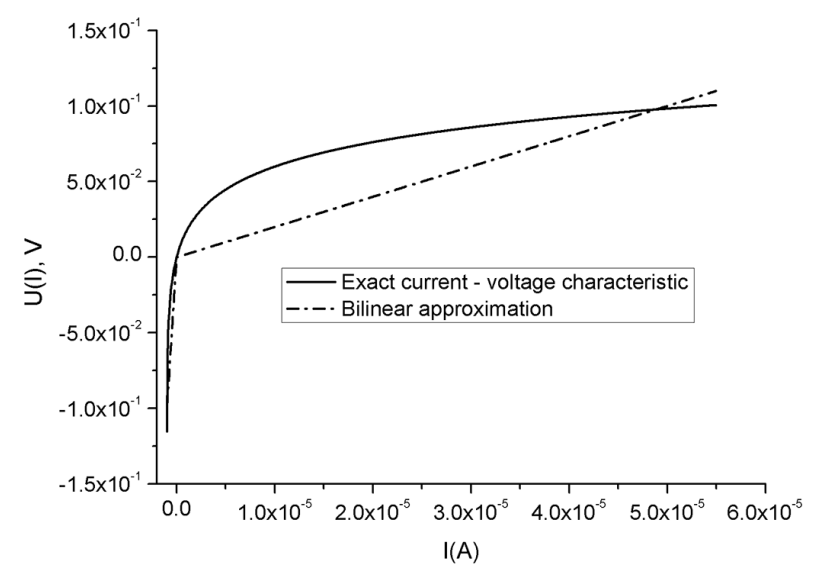

Figure 7. Characteristic of a generic diode and bi-linear approximation.

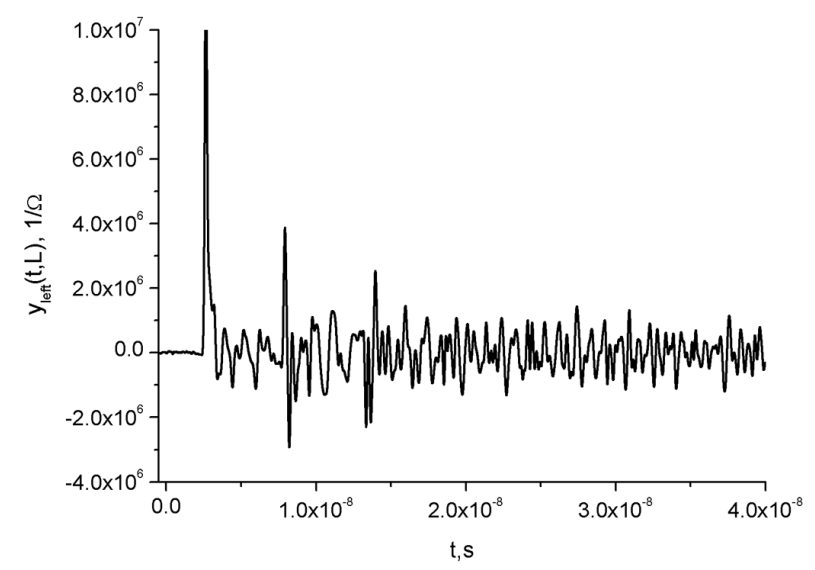

Figure 8. Left admittance function $\tilde{y}_{l}^{\left(Z_{C}\right)}(L, t)$.

One can recognize the first three peaks resulting from the exciting $\delta$-pulse along the TL. For $\tilde{y}_{l}^{\left(Z_{C}\right)}(L, t)$ the peaks are the first multiples of $L / c_{0}$. As expected, for $\tilde{y}_{r}^{\left(Z_{C}\right)}(L, t)$ the first peak is at $t=0$ and afterwards at time steps of $2 L / c_{0}$. The excitation of the TL gets phased out for increasing time steps as can be seen in Fig. 9. Because the left- and right-hand Green's functions (admittance functions) are known, the current at the end of the TL can be calculated provided that the excitation $u_{0}(t)$ is known. The following two tone excitation is used:

$u_{0}(t)=U_{0}\left[\sin \left(2 \pi f_{1} t\right)-\sin \left(2 \pi f_{2} t\right)\right] h(t) h\left(t_{0}-t\right)$

In Eq. (39) the Heaviside function is depicted by $h(t)$ and the following parameters are used: $U_{0}=1 \mathrm{~V}, f_{1}=300 \mathrm{MHz}$, $f_{2}=310 \mathrm{MHz}-400 \mathrm{MHz}, t_{0}=200 \mathrm{~ns}$.

Figure 10 shows the current at the end of the TL for the linear case with matched load. The frequency spectrum of the current is depicted in Fig. 11 where the two peaks at $f_{1}$ and $f_{2}$ can be clearly seen.

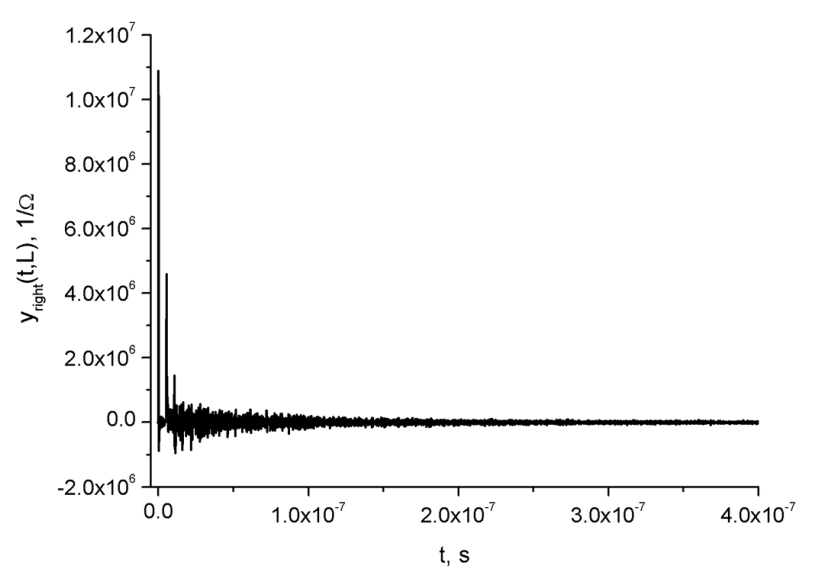

Figure 9. Right admittance function $\tilde{y}_{r}^{\left(Z_{C}\right)}(L, t)$.

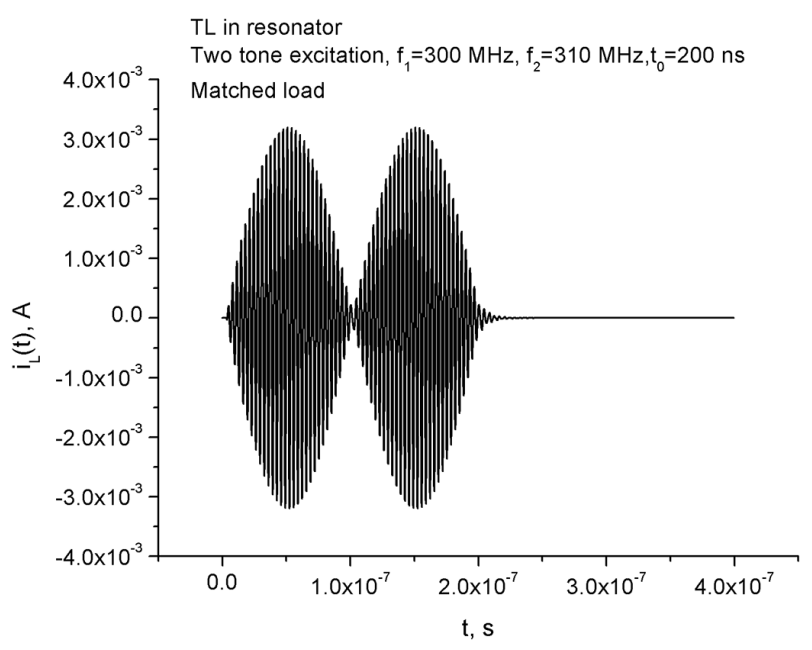

Figure 10. Current at the end of the TL in time domain (TL with linear and matched load in the rectangular resonator).

In Fig. 12 the current at the end of the TL in time domain is shown for the nonlinear load defined in Eq. (38). The second frequency $f_{2}$ of the two tone excitation was increased from $310 \mathrm{MHz}$ to $400 \mathrm{MHz}$ in steps of $10 \mathrm{MHz}$. The expected result can be seen in the frequency domain representation of the current at the end of the TL in the resonator. Figure 13 shows the result for the lowest frequency $f_{2}=310 \mathrm{MHz}$ and therefore for the lowest frequency difference $\Delta f=f_{2}-f_{1}=$ $10 \mathrm{MHz}$. Figures 14 and 15 show the currents in frequency domain for $f_{2}=350 \mathrm{MHz}$ and $f_{2}=400 \mathrm{MHz}$, respectively. The expected results are clearly observable: At the difference of the two tone frequencies, peaks occur which are not present in the linear case. Additionally, in contrast to the linear load, now there are peaks at multiples of the two basic frequencies $f_{1}$ and $f_{2}$. Around these multiples there are smaller peaks representing multiples of $\Delta f=f_{2}-f_{1}$. A moderate increase in peak heights with increasing $\Delta f$ is observable. 


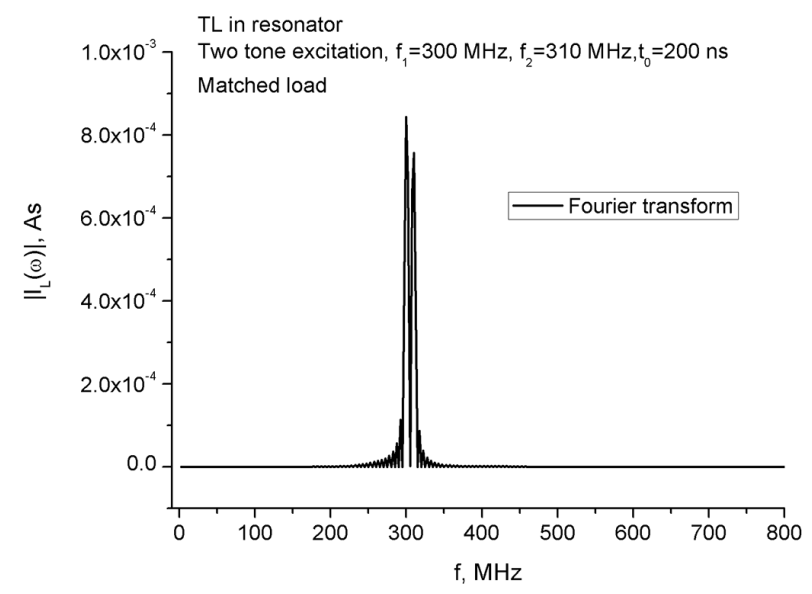

Figure 11. Current at the end of the TL in frequency domain (TL with linear and matched load in the rectangular resonator).

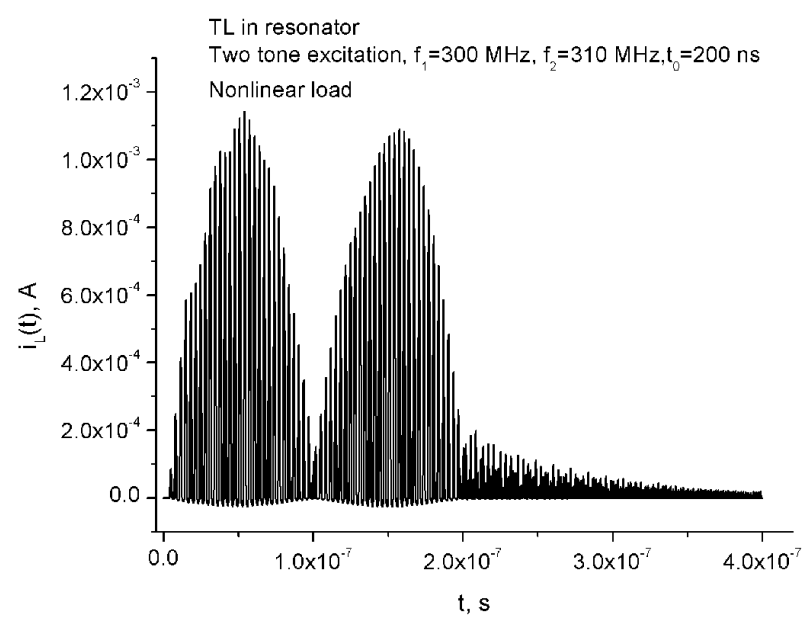

Figure 12. Current at the end of the TL in time domain (TL with nonlinear load in the rectangular resonator).

However, it must be stated that the known phenomena (by measurement) of significant increase in current amplitude for excitation frequencies corresponding to one or more eigenfrequencies of the resonator are not seen in the presented simulations. Also the used $\Delta f$ values do not correspond to the eigenfrequencies of the resonator. The reasons are: (a) The used resonator is quite flat and still behaves a bit like a waveguide. Additionally the TL has a high symmetry in the resonator. (b) Surely, the main reason is that the exciting frequencies were chosen too low. This had to be done because of performance limitations of the used standard computer. Going to higher excitation frequencies, the time steps have to be made smaller in time domain and the $\delta$-function has to be defined narrower. In almost the same manner, the frequency steps have to be made smoother in frequency domain calculations. A loophole would be the use of high-performance computers or a modified solution using methods of perturbation theory.

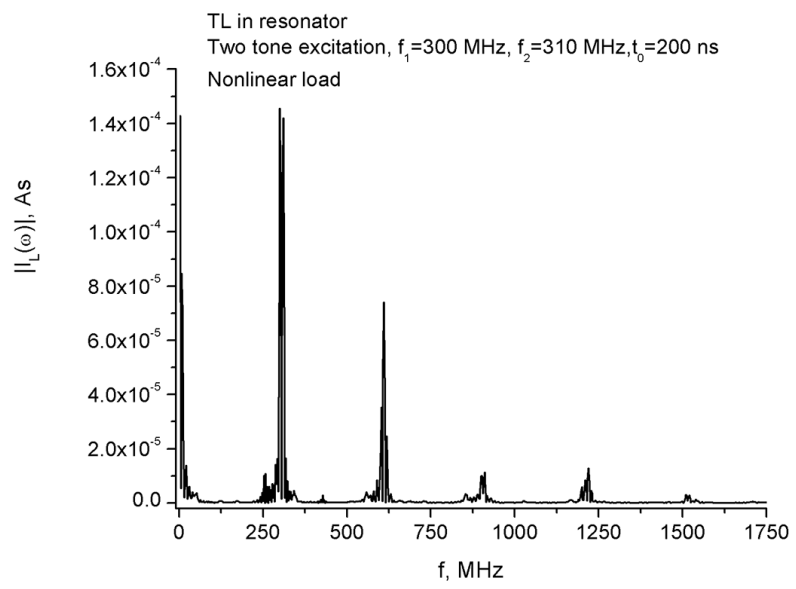

Figure 13. Current at the end of the TL in frequency domain (TL with nonlinear load in the rectangular resonator, $f_{2}=310 \mathrm{MHz}$, $\Delta f=10 \mathrm{MHz}$ ).

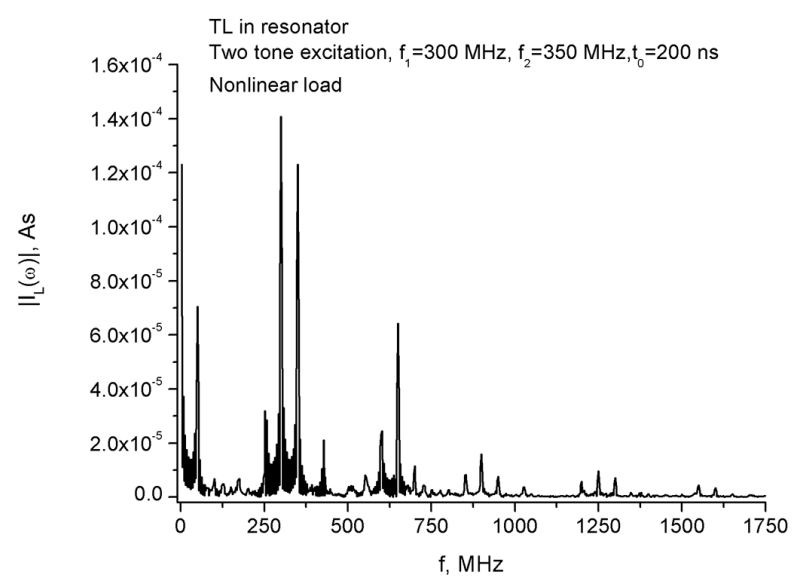

Figure 14. Current at the end of the TL in frequency domain (TL with nonlinear load in the rectangular resonator, $f_{2}=350 \mathrm{MHz}$, $\Delta f=50 \mathrm{MHz})$.

So far there is no comparison of the presented analytical results with those obtained by other methods. However, for the simple example of linearly loaded transmission lines in free space a lot of comparisons with other methods (e.g. Method of Tesche, Reflexion Method) have been done and an excellent agreement was observed (Tesche et al., 1997).

\section{Conclusion}

In this work nonlinearly loaded lossless transmission lines inside a rectangular resonator were studied. Using the leftand right-hand Green's functions of the problem in time domain an analytic solution could be presented. The Green's functions were developed for linear matched loads ensuring short duration of a pulse-shaped excitation in time domain and therefore acceptable calculation time. An example was presented using a simplified diode as nonlinear load and 


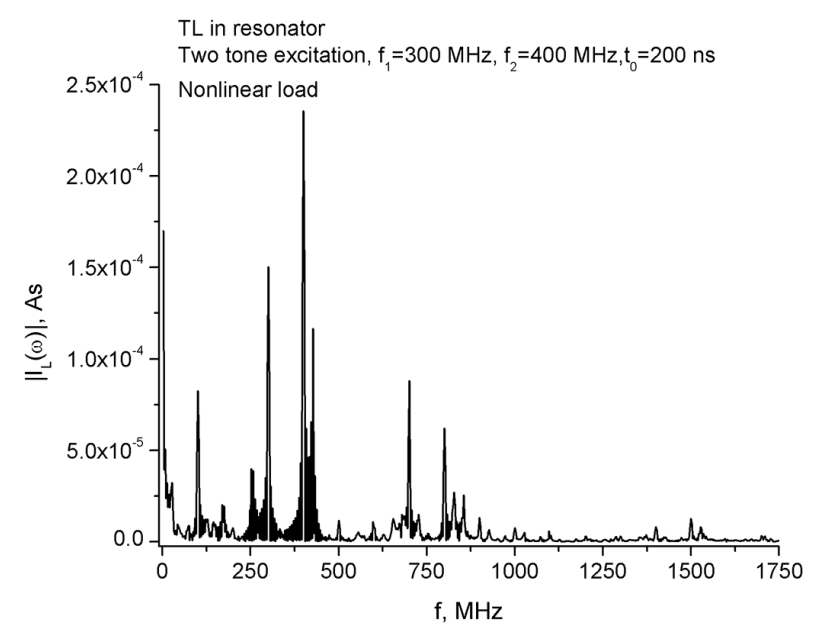

Figure 15. Current at the end of the TL in frequency domain (TL with nonlinear load in the rectangular resonator, $f_{2}=400 \mathrm{MHz}$, $\Delta f=100 \mathrm{MHz}$ ).

two tone excitations. The demodulation and intermodulation effects could be clearly demonstrated. At multiples of the excitation frequencies and their sums and differences distinct resonances appeared. It is worth mentioning that the method presented above can be extended to non-uniform multiconductor transmission lines with weak losses. This extension may be performed on the basis of the two publications (Rambousky et al., 2014) and (Tkachenko et al., 2014) and references given therein.

Edited by: F. Sabath

Reviewed by: H. Garbe and two anonymous referees

\section{References}

Djordjevic, A., Sarkar, T., and Harrington, R.: Analysis of lossy transmission lines with arbitrary nonlinear terminal networks, IEEE T. Microw. Theory, 34, 660-666, 1986.

Krauthäuser, H., Tkachenko, S., and Nitsch, J.: The action of nonlinear effects in a resonator, in: XXVIIth General Assembly of the International Union of Radio Science, p. 2167, Maastricht, 2002.

Liu, T. and Tesche, F.: Analysis of antennas and scatterers with nonlinear loads, IEEE T. Antenn. Propag., 24, 131-139, 1976.

Rambousky, R., Tkachenko, S., and Nitsch, J.: Calculation of currents induced in a long transmission line placed symmetrically inside a rectangular cavity, in: IEEE International Symposium on Electromagnetic Compatibility (EMC), 796-801, Denver, 2013.

Rambousky, R., Nitsch, J., and Tkachenko, S.: Transmission-Line Super Theory as Antenna Theory for Linear Structures, in: UltraWideband, Short-Pulse Electromagnetics 10, edited by: Sabath, F. and Mokole, E., Springer, Berlin, 2014.

Tesche, F., Ianoz, M., and Karlsson, T.: EMC Analysis Methods and Computational Models, John Wiley \& Sons, Inc., New York, 1997.

Tkachenko, S., Nitsch, J., and Rambousky, R.: Electromagnetic Field Coupling to Transmission Lines Inside Rectangular Resonators, Interaction Notes, Note 623, http://www.ece.unm.edu/ summa/notes/In/IN623.pdf, 2011.

Tkachenko, S., Rambousky, R., and Nitsch, J.: Electromagnetic Field Coupling to a Thin Wire Located Symmetrically Inside a Rectangular Enclosure, IEEE Trans. on EMC, 55, 334-341, 2013.

Tkachenko, S., Nitsch, J., and Rambousky, R.: Electromagnetic Coupling to Transmission Lines with Symmetric Geometry Inside Rectangular Resonators, in: Ultra-Wideband, Short-Pulse Electromagnetics 10, edited by: Sabath, F. and Mokole, E., Springer, Berlin, 2014. 